\title{
Understanding the process of living as signified by myocardial revascularization surgery patients
}

\author{
Alacoque Lorenzini Erdmann ${ }^{1}$ \\ Gabriela Marcellino de Melo Lanzoni ${ }^{2}$ \\ Giovana Dorneles Callegaro ${ }^{3}$ \\ Maria Aparecida Baggio ${ }^{4}$ \\ Cíntia Koerich $^{5}$
}

Objective: To understand the meanings for the process of living, for patients undergoing myocardial revascularization surgery, and to construct an explanatory theoretical model. Method: Grounded Theory was used, with data collection undertaken between October 2010 and May 2012, in a health institution which specializes in cardiac surgery, located in the south of Brazil. Thirty-three subjects were interviewed (patients, health care professionals and family members), distributed in four sample groups. Result: The explanatory theoretical model was comprised of 11 categories and the central phenomenon. The specialized service and the cardiac rehabilitation program formed the context, the discovery of the cardiac disease and the feelings experienced during the perioperative period were the cause and intervening conditions in the process of experiencing the myocardial revascularization surgery. The strategies were relying on the family's support, having faith and hope, and participating in the rehabilitation program. This process's main consequences were the confrontation of the changes and the resulting limitations, difficulties and adaptations to the new lifestyle after surgery. Conclusion: The process of experiencing the myocardial revascularization surgery constitutes an opportunity for maintaining the patient's life associated with the needs for confronting the significant changes in lifestyle.

Descriptors: Thoracic Surgery; Nursing; Myocardial Revascularization; Pain.

\footnotetext{
${ }^{1}$ PhD, Full Professor, Departamento de Enfermagem, Universidade Federal de Santa Catarina, Florianópolis, SC, Brazil.

2 Doctoral student, Universidade Federal de Santa Catarina, Florianópolis, SC, Brazil. Scholarship holder from Coordenação de Aperfeiçoamento de Pessoal de Nível Superior (CAPES).

3 MSc, RN.

4 Doctoral student, Universidade Federal de Santa Catarina, Florianópolis, SC, Brazil. Scholarship holder from Conselho Nacional de Desenvolvimento Científico e Tecnológico (CNPq), Brazil.

${ }^{5}$ Undergraduate student in Nursing, Universidade Federal de Santa Catarina, Florianópolis, SC, Brazil.
}

Corresponding Author:

Alacoque Lorenzini Erdmann

Universidade Federal de Santa Catarina. Departamento de Enfermagem

Rua Delfino Conti, s/n

Bairro: Trindade

CEP: 88040-370, Florianópolis, SC, Brasil

E-mail: alacoque@newsite.com.br 


\section{Introduction}

Diseases of the circulatory system have a high incidence in both the Brazilian and global context, being considered the principal cause of death and disability in Brazil and in the rest of the world(1). Treatment of cardiovascular diseases may be clinical or surgical, with surgical treatment considered an efficient option in treating ischemic cardiac diseases ${ }^{(2)}$ and indicated when it represents improved likelihood of survival for the patient $^{(3)}$. In addition to the increase in life expectancy, patients who undergo myocardial revascularization have a better evaluation of quality of life at six months after the surgical procedure(4).

The surgical procedure is sometimes perceived by the patient as an event related to incapacity and/ or alteration of body image ${ }^{(5)}$, and - because it is an atypical, stressful situation for the patients - makes them vulnerable, both in the pre- and post-operative periods, harming their recuperation. That this procedure has an important impact on the cardiac patient's process of living may be noticed through the doubts and uncertainties presented by the patients, regarding nutritional education, practice of physical exercise, returning to work, practice of daily activities, difficulties with the surgical incisions, practice of sexual activity, alcohol consumption, and use of medications ${ }^{(6)}$.

It is important for nursing to investigate the revascularized person's experiences and way of living, being healthy and falling ill, seeking to understand the meanings of the experiences lived through by the subjects, and to contribute with the care relationships, as they present a significant interface in adherence in self-care and an improvement in the process of rehabilitation ${ }^{(7)}$. Nursing can act by offering/exercising care which is personalized, creative and comprehensive, covering the totality and complexity of the human being ${ }^{(8)}$.

Based on the above, one may ask: how do the patients find meaning in their process of living after the experience of MRV surgery? Also, one can aim to understand the meanings about their process of living for patients who undergo MRV surgery, and to construct an explanatory theoretical model. Studies of this nature viabilize the comprehension of the surgical patient's experience, contributing to nursing care, teaching and research. Above all, it enables nursing to think/reflect on its care actions for cardiac patients, understanding them in their multiplicity of being and combining scientific knowledge with humane care actions.

\section{Method}

This study is qualitative and used Grounded Theory (GT) as its methodological framework, which allows it to explore the phenomenas' meanings, producing substantive theories which constitute guides for $\operatorname{action}^{(9)}$

The chosen setting was a health institution specialized in cardiac surgery, in the south of Brazil. The selection of the participants was intentional, based on the study's objective. For the first sample group, the inclusion criteria were: patients over 18 years of age, who had undergone MRV in the institution specialized in cardiac surgery at least thirty days previously. Data collection was undertaken in semi-structured individual interviews, digitally recorded, in the institution itself or in the participants' homes, between October 2010 and May 2012. The initial question was: Tell me about your experience of having undergone MRV surgery. The administering of the other questions was directed by the researchers, based on the interviewees' responses.

Thirty-three subjects participated in the study, being divided into 4 sample groups. The first group was comprised of 8 patients, who gave an account of the importance of the presence of health care professionals and family members in the perioperative process. The second group was composed of 2 nurses, one from the intensive care unit (ICU) and the other from the coronary inpatient unit; 2 doctors, one working in the medical specialties outpatient clinic, and the other in ICU; physical education teacher and one nurse technician, both assigned to the rehabilitation service. The third group was composed of 4 patients and 5 family members (direct carers). The fourth group was made up of another 10 patients, with a view to confirming the hypotheses raised. Theoretical saturation was reached at this point.

The data was collected and analyzed simultaneously, following the stages proposed by GT: open, axial and selective coding. In open codification, the data was analyzed line by line, with the objective of identifying each incident, forming the preliminary codes. In the axial codification, the data was grouped, with a view to relating categories to their subcategories, so as to obtain a clearer and more complete explanation of the phenomenon, as well as of its properties and dimensions. In the phase of selective codification, the categories and subcategories found previously were compared and analyzed continuously, with the objective of integrating and refining the theory, in this way identifying the 
central category. Next, the theoretical explanation of the categories was developed, according to the paradigmatic model(9). Complexity Theory was used as the theoretical framework ${ }^{(8)}$.

Eleven categories emerged from the process of analysis, which, inter-related, underpin the phenomenon "Perceiving the process of experiencing MRV surgery as an opportunity for maintaining life, associated with confronting significant changes in lifestyle". The validation of the Theoretical Model was implemented by a patient (who was not a deponent) who had undergone MRV surgery in the institution, and by a researcher - a nurse - with expertise in the method.

Ethical aspects were respected throughout the different stages of the research(8). To guarantee the participants' anonymity, it was decided to use the initials ' $E$ ', ' $P$ ', ' $F$ ' and 'PA', which identified, respectively, the first, second, third and fourth sample groups, followed by ordinal numbers, according to the order of the statements.

\section{Results}

The phenomenon "Perceiving the process of experiencing MRV surgery as an opportunity for maintaining life, associated with confronting significant changes in lifestyle" is comprised of 11 categories, organized in 5 components, in accordance with the paradigmatic model(9), shown in graphical form in Figure1.

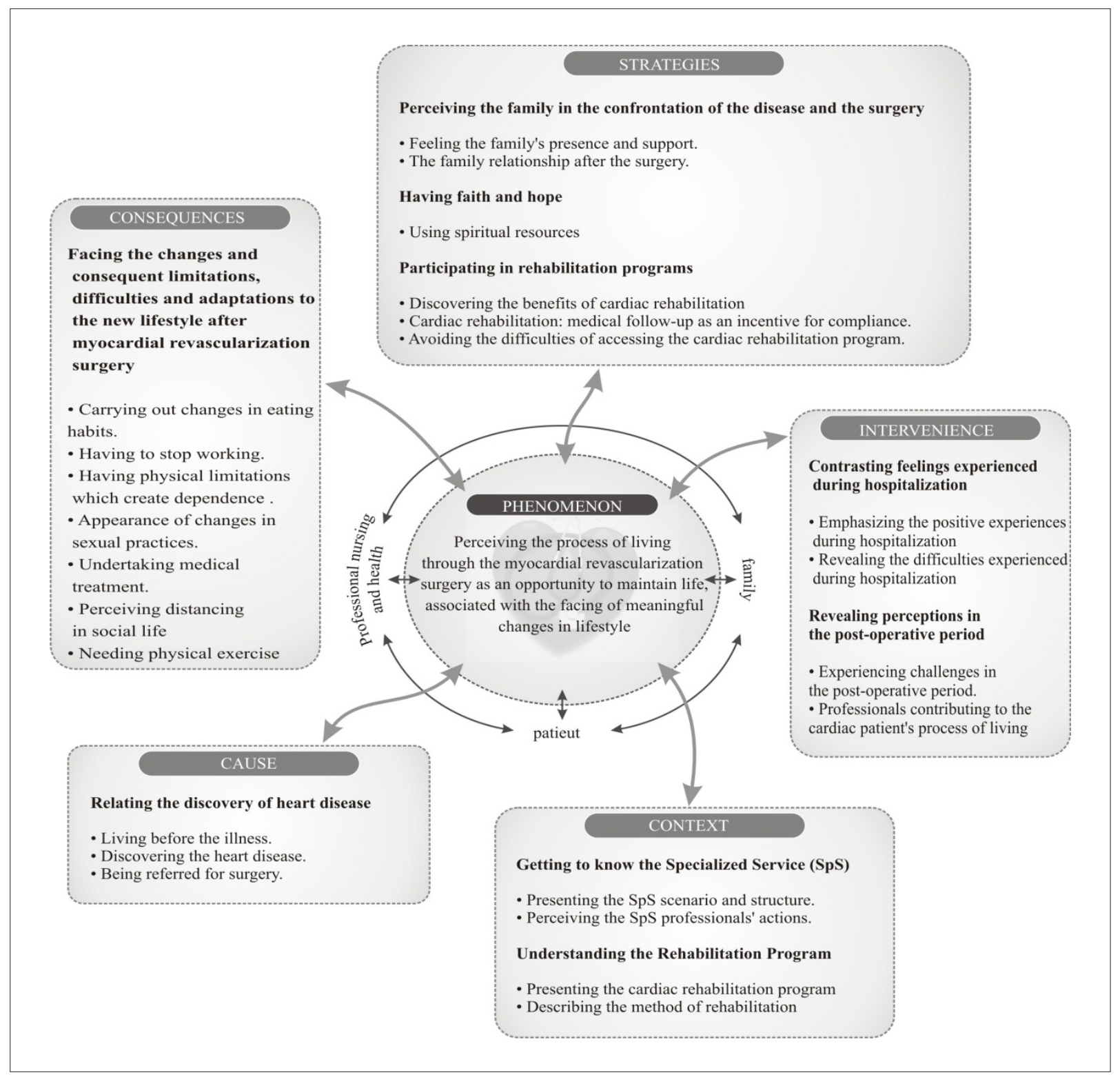

Figure 1 - Diagram of the phenomenon of the study, and its categories 


\section{Context}

The categories "Getting to know the specialized service" and "Understanding the rehabilitation program" present the context in which the phenomenon takes place. The hospital is valued for being a center of excellence in cardiology, which carries out transplants and major surgery, although it has weaknesses of various types. Among the weaknesses, the lack of beds, equipment and staff all stand out, leading to queues and significant impacts for the patient, as one health care professional's statement describes: The reason for the slowness in attending patients is the shortage of beds, not just coronary unit ICU beds but beds in the department. [...] The structure should be scrapped. A lot of things are lacking, of which the most important is people - we're short of staff. (P2)

The intensive care unit (ICU) was mentioned by the participants as a cruel scenario. In the first hours after the MRV surgery, on waking, the patient sees that he or she has the orotracheal tube in place, has drains and pains, and identifies noises which worsen the feeling of discomfort. Sleep and relaxation are impeded by the constant presence of health care professionals, due to the frequent monitoring and administration of medication.

For accompanying the patients in their recuperation, the institution has a cardiac rehabilitation program, set up in 1997, with the objective of supporting the undertaking of physical activities, given that that not all State primary health care centers offer this service. This program has gradually been valued and has received improvements by the institution, as evidenced through the increase of the physical structure and the amount of equipment. The time taken for rehabilitation by each patient is respected by the health professionals, and there are patients who have been linked to the program for years.

\section{Causal Condition}

The phenomenon is initiated by the category "Reporting the discovery of the heart disease", which covers from the first signs and symptoms of the acute myocardial infarction up to the experience of the surgical process. The participants report that before the surgery they had active lives, with an overload of work, the frequent habit of eating meals rich in saturated and animal fats (red meat) and consumption of alcohol and/ or tobacco, associated with sedentarism and failure to carry out regular health check-ups.

The discovery of the heart disease was not a surprise for the majority, as they were already aware of the presence of hereditary factors. The initial manifestations, such as pain in the arm or chest, dizziness, sudoresis and the feeling of imminent death caused the participants to go to various health services: Local Healthcare Units, Emergency Care Units and Emergency Departments.

Although all the patients had undergone MRV, some, initially, had undergone cardiac catheterization followed by angioplasty, with the placement of stents, while others had been sent for revascularization as a priority intervention. Associated with the physicians' reports about the need for surgery, some participants refer to the wish to undergo surgery to save their lives, as shown in this account: I had a $1 \%$ chance of survival. [...] When the time came for me to go for the surgery, hat gurney was happiness. It seems I was changing my life. (E3)

The health team, in particular the nurses, places special attention on pre-operative counseling for patients and family members through group and/or individual encounters, aiming to minimize the stress of hospitalization and the process of surgery and to reduce the anxiety and the possible complications.

\section{Intervening Conditions}

The conditions which interfere in the process of living of the patients undergoing MRV are presented in two categories. In the first category, "Contrasting feelings experienced during the hospitalization", it is revealed that the fear of death and of anesthesia, the risks posed by surgery and the separation from the family act as a barrier to accepting the surgical procedure. Some patients felt sad, desperate, or with a constant desire to cry, because of not wanting to undergo the procedure. However, others received the news very calmly, priding themselves on having the courage to undergo the surgical procedure.

The participants emphasized the competency of, and the good interpersonal relationship with, the health team, and stressed their gratitude for the direct care and for the emotional support, and confidence.

The participants considered the wait for surgery an obstacle in the process of living of the patients undergoing MRV, as waiting for the surgery increases the anxiety and may cause worsening of the clinical condition of health. Some patients waited uncomfortably in beds in the corridors of the Emergency Department for spaces in the inpatient unit, and later for a space in ICU, increasing even further the fear of not surviving the surgical procedure.

In the second category, "Revealing the perception in the post-operative period", the first hours after 
the surgery are described. The patients, on waking, feel disoriented in time and space, feel discomfort because of the constantly-on lights, and distressed by the impossibility of speaking due to the presence of the orotracheal tube, felt pain and discomfort during extubation and pain in the thoracic incision every time they coughed.

MRV raises questions about the future, such as inability to work, the occurrence of retirement and the reduction in family income. The nursing professional is understood as a foundation, who gives attention, guides and manages the necessary challenges, as the following accounts illustrate: They cling to us, if you give real attention, they want that person who they trust. (P3) It was difficult to go home. Here, I had nurses, doctors, everything around me. At home it was just me and my husband. (PA4)

The health professionals point to the continuity of treatment as an obstacle, as they know the difficulties in arranging appointments with the health professionals in Primary Care, and the difficulty in acquiring medication with one's own resources, when these are not available through the Unified Health Service (UHS).

\section{Strategies}

Three categories emerged from the analysis, which comprise the strategies used for the achievement of the phenomenon. The category "Perceiving the family in the confrontation of the disease" describes how the family is involved by the health care professionals in preand post-operative counseling meetings, the family's importance being emphasized in the accompaniment of the patient, in the offering of support and security, and the reduction of the anxiety and of the stress of surgery.

After discharge - although the care and cooperation from the family benefit the patient's recuperation emotional harm is caused by situations of family conflict in family and social interaction, in particular by family members' demands that patients change their lifestyles. However, the importance of the patient's autonomy in selfcare can be ascertained, as in this statement from a health care professional: We encourage them to take control too, also in the process of treatment and secondary prevention. (P5)

The category "Having faith and hope" indicates the patients' use of spiritual resources as a source of hope and for facing the process of surgery. The health care professionals assert that they incentivize the use of this support.

The category "Participating in the rehabilitation program" discusses the patients' initiation in the cardiac rehabilitation program while still receiving inpatient treatment, informing about the advantages of participating in the program, such as the facilitated medical follow-up, and the involvement of the family in the process.

Participation in the cardiac rehabilitation is compromised by the unfavorable economic situation of some patients, who due to living far away and depending on transport, abandon the program. In these cases, the patient is advised to participate in other groups which work with physical reconditioning, bearing in mind, however, the cardiac surgery.

\section{Consequences}

The category "Facing the changes and consequent limitations, difficulties and adaptations to the new lifestyle after MRV surgery" emphasizes the need for the patient to stop working, one of the first and hardest decisions to be taken. Many associate the fact of having to stop working as if they were taking their own lives, as in the statement: I started crying, if you take this from me (work), you're taking away my life, because I have always been accustomed to work. (E2)

The alteration of occupational routine affects the patient's emotional aspect and also the patient's economic and financial condition, as, often, the patient is the family breadwinner. According to the patients' accounts, the family budget is affected, and the compliance with treatment compromised, by the unavailability of medication(s) for cardiac treatment on the UHS and the need for the patients to acquire them out of their own pockets.

The patients emphasize the challenge of getting their sex lives back after surgery. The changes, however, can be overcome when the partner understands the needs and the new possibilities for obtaining pleasure.

With guidance and follow-up from the health team, the patient understands that physical activity is a tool for overcoming problems, facilitative and beneficial to their living well. By contrast, the patients point to the idea of privation and of limitation of habitual activities, held as pleasurable, but harmful to the health. In relation to the social aspect, the families gradually notice the separation of the individual who has undergone MRV from family conviviality, from children, grandchildren and friends.

\section{Discussion}

Understanding that human beings are complex and suffer interference from movements/actions/influences 
from their network of relationships and interactions ${ }^{(8)}$, one can detect how significant the process of MRV is, observing also its impact on the social and economic spheres.

The excessively-lengthy waiting period for the surgery, which creates anxiety and discomfort for the patient and their family, according to research undertaken in a Rio de Janeiro public teaching hospital, may be explained by the lack of beds in the post-operative phase, a condition which explains the postponing of cardiac surgery and, consequently, an increase in the patients' time of hospitalization(10). This fact compromises the daily institutional dynamic, results in higher public costs, and increases the risk of acquiring a nosocomial infection in the patient awaiting surgery ${ }^{(10)}$.

The rates of anxiety and depression in patients in the pre-operative phase for cardiac surgery are high, especially among the elderly, considering that prolonged hospitalization encourages these emotional disorders. Anxiety tends to increase the pain rating score and, consequently, the use of analgesia in the post-operative phase ${ }^{(11)}$. In the post-operative phase the patients encounter an intensive care environment (ICU), which due to its rigid and inflexible routines entails disturbance of sleep and loss of privacy and autonomy for the patient ${ }^{(12)}$.

Because the patients report pain in the immediate post-operative period, probably due to the insertion of drains, the surgical incision and the cough, it becomes necessary to administer opioids and analgesics for pain control, for the patient's comfort and for the reduction of physiological changes resulting from the pain, such as the elevation of blood pressure and respiratory frequency ${ }^{(13)}$. The nursing professionals can work attentively to ensure care which manages to take account of the human being's multiple dimensions and needs, reflecting on physical, emotional and relational needs, among others, with the aim of making the environment and the experience less traumatizing(8).

It is observed that cardiac rehabilitation initiated in the pre-operative period, as well as reducing levels of post-operative complications and consequently reducing the duration of hospitalization, allows the patient to be more prepared to face the surgery ${ }^{(12)}$.

The patients in this study relate the appearance of cardiovascular disease to stressful lifestyles and to living habits considered unhealthy. These conditions were verified in a similar research project, in which the patients describe and explain the following as principal factors for the appearance of heart disease: stress, the chronic underlying diseases, such as high blood pressure and diabetes, inadequate eating habits (such as consumption of fat), smoking, and sedentarism(14). It can be verified that the control of these risk factors for cardiovascular disease is important for the prevention of the disease ${ }^{(15)}$.

According to research carried out in the Cardiac Emergency Unit in Pernambuco it is confirmed that, irrespective of being the first episode of heart attack or of being a new event, the patients' immediate response to the symptoms - taking analgesics and resting - is inadequate, as it postpones contact with the emergency services. Such an attitude reveals a lack of recognition of the disease's clinical manifestations and tends to worsen the intrahospital outcomes for the patient ${ }^{(16)}$.

The health team's care, whether clinical or interactive, is considered essential for the cardiac patient. From the perspective of complexity, the care is understood as a process of interactions and associations between beings, making connections possible based on subjective forces between the cared-for being and the caregiver, being part of the health system, dynamizing it, organizing it and co-organizing it, along with the other social systems ${ }^{(8)}$. In addition to the professional care received, spirituality is perceived as having a supporting role, a support used by the cardiac patients in the face of challenges in moments of crisis, as is the case with heart disease and the surgical process ${ }^{(17)}$.

Participation in the cardiac rehabilitation program after discharge from hospital, in addition to contributing to the maintenance of the patient's physical condition, benefits his or her social interaction, reducing the rates of depression and anxiety after MRV surgery(18). The rehabilitation groups provide motivation for the change and are important for the evolution and continuity of the educational process.

The patients, however, frequently have difficulties in keeping up the care recommended by the health care professionals for preventing cardiovascular events. Compliance with the care is influenced by the meaning attributed by patients to health, heart disease and personal risk, as well as by socioeconomic status, motivation and desire for change, self-efficiency, and by sources of health information considered reliable, sendo constituída de uma relação concordante between health care professional and patient by means of da da use of appropriate tools in the interactive process ${ }^{(19)}$.

It is noteworthy that a study undertaken with heart disease patients for evaluating the effectiveness of a nursing educational program on the improvement of self- 
care behaviors in patients with heart failure determined that educational gatherings, home visits, tele-nursing and printed booklets have a beneficial effect on the process of compliance and maintenance of treatment ${ }^{(20)}$. Thus, investment in innovation and improvement of care strategies for the nursing team to follow up heart disease patients over time becomes essential.

The patients avoid talking about sex and mention fear in relation to sexual practices after the surgery. The spouses normally understand their partner's fragility after the cardiac event and act with more delicacy or respect, but nevertheless it is confirmed that after the cardiac surgery, the frequency of sexual relations is reduced ${ }^{(21)}$. It is necessary to discuss the cardiac patient's sexuality, using the appropriate language and creating suitable settings for the discussion. Continuing education for health care professionals, as well as research about sexual counseling in the post-operative period are necessary for the evaluation of problems in this matter and for appropriate and timely counseling of cardiac patients and their spouses ${ }^{(22)}$.

\section{Conclusion}

This study's results evidenced the phenomenon "Perceiving the process of living through MRV surgery as an opportunity to maintain life, associated with the facing of meaningful changes in lifestyle" based on the inter-relation of eleven categories found and on the paradigmatic theoretical model constructed.

Regarding the study's limitations, it stands out that the data collection was undertaken only with patients and professionals from an institution specialized in cardiac surgery, which offers outpatient follow-up with a structured rehabilitation program. The production of further studies is therefore suggested, with individuals who have undergone MRV in other institutions and who are not in a rehabilitation program, with a view to identifying other specificities.

The process of living through MRV surgery constitutes an opportunity for maintaining the life of the patient, associated with the needs for facing the significant changes in lifestyle. Both submitting to complex surgical treatment and needing to change one's lifestyle in order to maintain one's life are very strong states and conditions experienced by people who are physically and emotionally weakened by ischemic heart disease. This study's results indicate important meanings to be considered in the nursing care for people undergoing MRV surgery, principally regarding the maintenance of friendly, trusting and emotionallysupportive care relationships to overcome family absences and changes in lifestyle. Further, it stands out that the process of health education must be a constant in the process of living of patients who have undergone MRV, with a view to the maintenance of, and motivation for self-care and healthy living habits.

\section{References}

1. Ramos GC. Aspectos relevantes da doença arterial coronariana em candidatos à cirurgia não cardíaca. Rev Bras Anestesiol. 2010;60:662-5.

2. Botega FS, Cipriano JG, Lima FV, Arena R, Fonseca JH, Gerola LR. Cardiovascular behavior during rehabilitation after coronary artery bypass grafting. Rev Bras Cir Cardiovasc. 2010;25(4):527-33.

3. Pivoto FL, Lunardi WD Filho, Santos SSC, Almeida MA, Silveira RS. Nursing diagnoses in patients in the postoperative period of cardiac surgery. Acta Paul Enferm. 2010;23(5):665-70.

4. Gois CFL, Dantas RAS, Torrati FG. Qualidade de vida relacionada à saúde antes e seis eses após a revascularização do miocárdio. Rev Gaúcha Enferm. 2009 dez;30(4):700-7.

5. Souza NVDO, Silva MF, Assumpção LR, Nunes KSM, Morgado FM, Amorim LKA. Perfil dos clientes que receberam orientações sobre cuidados perioperatórios. Rev Enferm Atual. 2008;43(8):34-7.

6. Almeida PFP, Góes-Júnior R, Gasparino RC. Dúvidas dos pacientes em pós-operatório de revascularização do miocárdio. Cogitare Enferm out/dez 2009;14(4):675-81. 7. Andrade ACV, Soratto MT. Education in Health for Post-Cardiac-Surgery Patients' Self Care. Saúde Rev. 2009;11(28/29):37-47.

8. Baggio MA, Erdmann AL. Teoria fundamentada nos dados ou Grounded Theory e o uso na investigação em Enfermagem no Brasil. Rev Enferm Referência 2011;3(3):177-85.

9. Nascimento KC, Erdmann AL. Understanding the dimensions of intensive care: transpersonal caring and complexity theories. Rev. Latino-Am. Enfermagem. 2009;17(2):215-21.

10. Chaves Sá SP, Gomes do Carmo T, Secchin Canale L. Evaluating the performance indicator surgical suspension as a factor in the quality of care to the patient. Enferm Global. 2011;10(23):200-9.

11. Jack S, West M, Grocott MP. Perioperative exercise training in elderly subjects. Best Pract Res Clin Anaesthesiol. 2011;25(3):461-72. 
12. Baggio MA, Pomatti DM, Bettinelli LA, Erdmann AL. Privacy in critical care units: the patient's rights and implications for nursing professionals. Rev Bras Enferm. 2011;64(1):25-30.

13. Andrade EV, Barbosa M, Barichello E. Pain assessment in postoperative cardiac surgery. Acta Paul Enferm. 2010;23(2):224-9.

14. Vila VSC, Rossi LA, Costa MCS. Heart disease experience of adults undergoing coronary artery bypass grafting surgery. Rev Saúde Pública. 2008;42(4):750-6. 15. Scherr C, Ribeiro JP. Chemical food composition: implications for atherosclerosis prevention. Rev Assoc Med Bras. 2011;57(2):153-7.

16. Gouveia VA, Victor EG, Lima SG. Pre-hospital attitudes adopted by patients faced with the symptoms of acute myocardial infarction. Rev. Latino-Am. Enfermagem. 2011;19(5):1080-7.

17. Guerrero GP, Zago MMF, Sawada NO, Pinto MH. Relationship between spirituality and cancer: patient's perspective. Rev Bras Enferm. 2011;64(1):53-9.

18. Blumenthal JA. New frontiers in cardiovascular behavioral medicine: Comparative effectiveness of exercise and medication in treating depression. Cleve Clin J Med. 2011;78 Suppl 1:S35-43.

19. Cohen SM. Concept analysis of adherence in the context of cardiovascular risk reduction. Nurs Forum. 2009 Jan-Mar;44(1):25-36.

20. Dalteg T, Benzein E, Fridlund B, Malm, D. Cardiac disease and its consequences on the partner relationship: A systematic review. Eur J Cardiovasc Nurs. 2011;10(3):140-9.

21. Rodriguez-Gazquez MA, Arredondo-Holguin E, Herrera-Cortes R. Effectiveness of an educational program in nursing in the self-care of patients with heart failure: Randomized controlled trial. Rev. Latino-Am. Enfermagem. 2012; 20(2):296-306.

22. Arenhall E, Kristofferzon M, Fridlund B, Malm D, Nilsson $U$. The male partners's experiences of the intimate relationships after a first myocardial infarction.

Eur J Cardiovasc Nurs. 2011;10(2):108-14. 